\title{
A FRAMEWORK FOR DATA TRAFFIC IN COGNITIVE RADIO NET- WORKS USING TRUSTED TOKEN ANALYZER SCHEME
}

\author{
Nikitha Lee Joy ${ }^{1}$, Sangeetha Senthilkumar ${ }^{2}$, S.Sathyaraj ${ }^{3}$ \\ ${ }^{I} P G$ Student, Oxford Engineering College, Trichy \\ ${ }^{2}$ Assistant Professor, Oxford Engineering College, Trichy \\ ${ }^{3}$ Assistant Professor, Oxford Engineering College, Trichy
}

\begin{abstract}
A cognitive radio network is considered as a key technology for future wireless communications. The motivation behind cognitive radio is the effective usage of limited spectrum. Cognitive radio networks are designed to opportunistically exploit the underutilized or unused spectrum bands. Cognitive radio combines sensing, learning, and optimization algorithms to manage and adapt the radio system from the physical layer and up the communication stack. Spectrum Sensing, Spectrum Decision, Spectrum Mobility and Spectrum Sharing, are the four functionalities involved in Cognitive Radio Networks. This paper proposes a framework for data traffic management by considering the QoS requirement of the secondary user and also the primary user activity. The Location information of the primary user in cognitive radio networks can be used to assist the communication among secondary users outside the transmission coverage area of primary users and also for tracking the primary user activities. The objective of the paper is to design a framework which will support the secondary user data transmission while considering the primary user activity and QoS requirement.
\end{abstract}

Keywords- Cognitive Radio Networks, Primary User Activity, QoS management, Trusted Token Analyzer

\section{INTRODUCTION}

Cognitive radio is a recent novel approach for the realization of intelligent and sophisticated wireless systems. Software Defined Radio, a recent development in wireless technology, promise to address some of the major limitations like inefficient utilization and management of the radio frequency (RF) in both licensed and unlicensed spectrum band experienced by legacy wireless communication system. A radio that is able to reliably sense the spectral environment over a wide bandwidth, detect the presence/absence of legacy users and use the spectrum only if communication does not interfere with any primary user is called a cognitive radio. The seminal paper of J. Mitola [8] introduced the concept of cognitive radio. Ian F. Akyildiz et al. [1] defines cognitive radio as: "A Cognitive Radio is a radio that can change its transmitter parameters based on interaction with the environment in which it operates".

Cognitive radio networks are composed of cognitive radio devices. An interconnected set of cognitive radio devices that share information is defined as a cognitive radio networks. (CRN).Cognitive radio networks (CRN) are envisioned to provide high bandwidth to mobile users via heterogeneous wireless architectures and dynamic spectrum access (DSA) techniques. Cognitive Radio Networks aim at performing the cognitive operations such as sensing the spectrum, managing available resources, and making user-independent, intelligent decisions based on cooperation of multiple cognitive nodes.
CR networks however impose unique challenges because of the high fluctuation in the available spectrum as well as the diverse quality-of-service (QoS) requirements of various applications and secondary users. To address these challenges, first, CR networks are required to determine which portions of the spectrum are available, called spectrum sensing [2], [10]. Furthermore, how to coordinate multiple CR users to share the spectrum band, called spectrum sharing, is another important issue in CR networks [6], [16].Secondary users have to vacate the channel for the primary user, when they arrive, called spectrum mobility is another aspect of CRN.Fig.1shows the dynamic spectrum management framework [2]. Spectrum decision in CRN refers to the ability of the secondary users to select the best available spectrum band to satisfy the user's quality of service requirements. Quality of Service for the secondary users involves data traffic management.

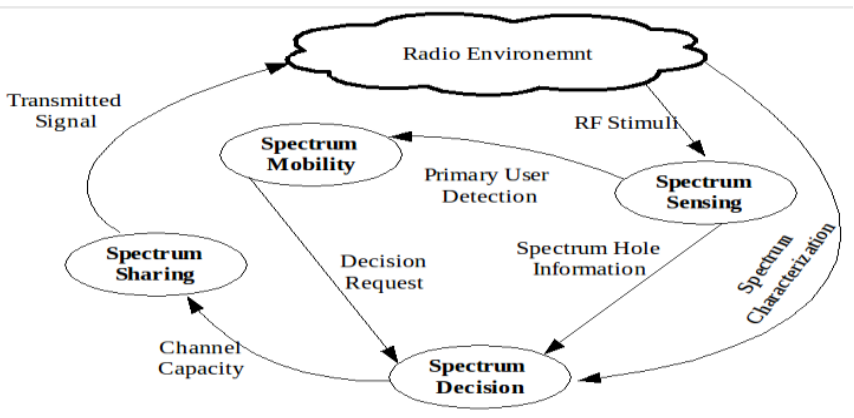

Fig 1: Dynamic Spectrum Management Framework 
To improve spectrum efficiency in cognitive radio networks, many operations such as sharing data in cooperative spectrum sensing, broadcasting spectrum-aware routing information, and coordinating spectrum access rely on control message are exchanged on a common control channel. Thus, a reliable and "always on" control channel is unavoidable in CRN.

\section{RELATED WORKS}

\subsection{Spectrum Decision}

For spectrum allocation, a global optimization scheme is developed based on graph theory [17]. Distributed spectrum allocation based on local bargaining is proposed in [4], where CR users negotiate spectrum assignment within local selforganized groups. In [20], a dynamic channel selection scheme is developed for delay-sensitive applications based on a priority queuing analysis and a decentralized learning algorithm.

\subsection{QoS Management /Data Traffic Management}

QoS support in CR systems is critical to ensure its success in consumer wireless market .In contrast to traditional wireless communication networks, one of the major challenges faced in cognitive radio networks is the interruptions from primary users. The service of packets in cognitive radio network has to be interrupted when primary users emerges. Usually, the emergence of primary user is assumed to be stationary., Such an assumption may not be true in practical means, since the traffic pattern of primary users could be time variant. [9] Specifies transient analysis of data traffic in cognitive radio networks.

A time-varying service capacity is discussed in [12] to widen the application. The problem of designing effective routing solutions for multi-hop CRNs, which is a focal issue to fully unleash the potentials of the cognitive networking paradigm, is focused in [25].

\section{PROPOSED SYSTEM}

\subsection{System Model}

Primary radio nodes are the legacy users and they can access their respective licensed bands without any restriction. Indeed, PR nodes have the highest priority to access the channels and should not be interrupted by the CR nodes.

We consider an OSS environment, where secondary users equipped with $\mathrm{CRs}$ are operating over $\mathrm{N}$ orthogonal frequency channels that are licensed to legacy(primary) users. One of the nodes is considered as head node or server. It involves the trusted token analyzer scheme and location verification schemes.

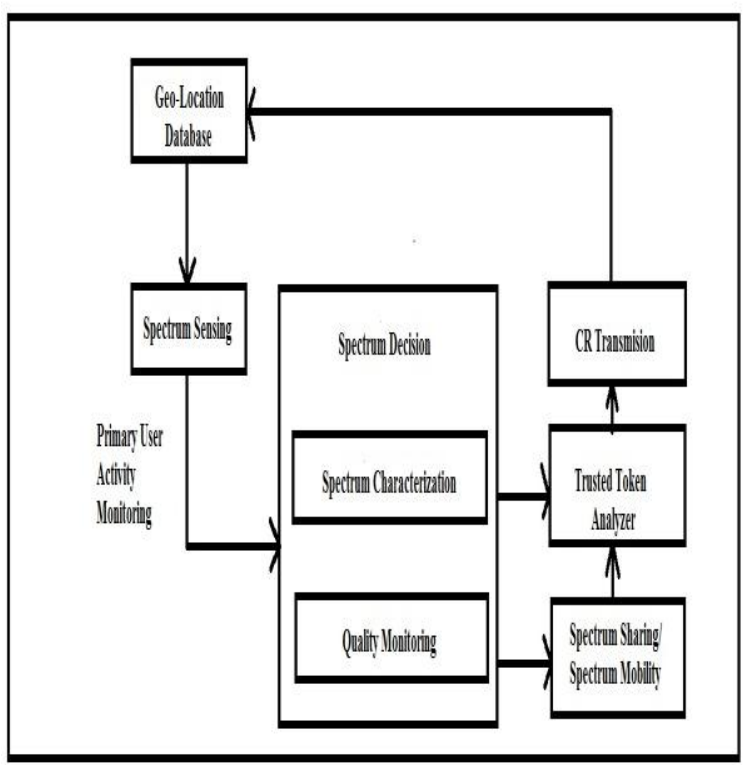

Fig 2: General Framework

In cognitive radio network each spectrum band is characterized based on local observations and on statistical information of the primary networks which is normally called PU activities. The selection of the most appropriate spectrum band is the next step in spectrum decision, based on the spectrum band characterization. Thirdly, a CR should be able to reconfigure its transceiver parameters to support communication within the selected spectrum band.

Once the spectrum decision is made, and a particular spectrum is chosen for secondary data transmission, the rendezvous process is initiated. Link establishment in distributed cognitive radio networks requires the communicating nodes to be rendezvous to exchange control

Figure 2 specifies the general frame work for CR transmission. Geo-location database contains the information like the channel condition, capacity of the channel etc. The primary user activity is monitored in spectrum sensing along with the sensing of unused or under-utilized spectrum. Based on these information the spectrum decision is made which involves the spectrum characterization and also the quality of the service and users quality requirement are monitored here. Based on the decision the channel is selected for CR transmission. 


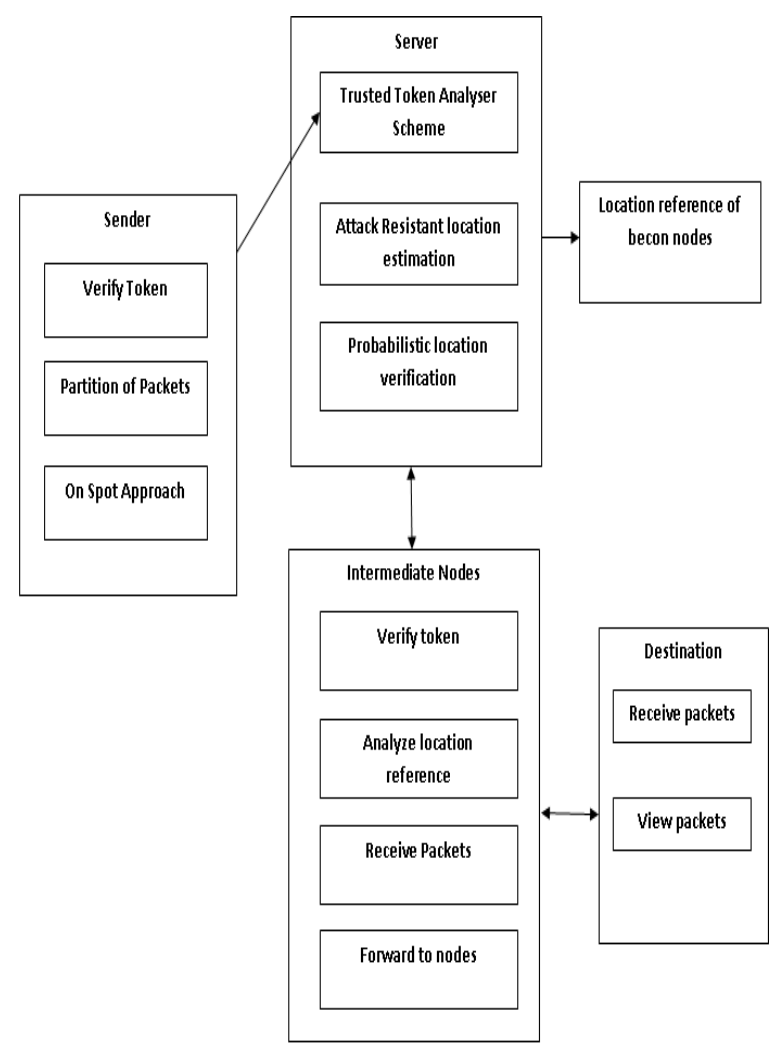

Fig 3: Trusted Token Analyzer

\subsection{Probabilistic Location Verification}

Location verification is an effective defense against attacks which take advantage of a lack, or compromise, of location information in the spectrum sensing. Beacon nodes[ Fig 3] will perform the spectrum observation. Beacon nodes will collect all the local information and the availability of the spectrum holes. Beacon nodes will hand over these local observations to the head node. Head node involves probabilistic location verification scheme which will in turn locate the location of the spectrum holes. The location of the identified spectrum hole is indeed verified in probabilistic location verification against fake spectrum hole information.

\subsection{Trusted Token Analyzer Scheme}

The general architecture for traffic management [fig 2] involves a server with trusted token analyzer, Attack resistant location estimation and location verification. Fig 3 gives the specific view of the trusted token analyzer scheme. Token bucket algorithm is used for generating tokens. The bucket represents a rate-limiting function of the policer on the interface input or output traffic .Each token in the bucket represents a "credit" for some number of bits, and tokens in the bucket are "cashed in" for the ability to receive or transmit traffic that conforms to a rate limit configured for the policer. The token arrival rate is the fixed bits-per-second rate at which tokens are added to the token bucket, but only up to the speci- fied depth of the bucket. Tokens are added based on the information from the quality monitoring and the primary user activity monitoring. If both are satisfied a token is added into the bucket. The token bucket depth defines the capacity of the bucket in bytes.CR transmission takes place only when there are sufficient tokens are available in the bucket, thus controlling or policing the traffic.

When the sender needs to send a packet to the receiver, it will confirm the server for the availability of enough tokens. If enough tokens are available then the sender will send the packet. If the packet is received by any intermediate node, then it will analyze the location reference and it will again confirm the traffic conditions to the server and will then forward the packet to the destination. Once the packet is received by the intended receiver, it can view the message by opening the packet.

\section{CONCLUSIONS}

Spectrum Sensing is performed to identify the spectrum availability. Each CR performs spectrum sensing to identify the available spectrum bands and the spectrum decision process selects from these available bands for opportunistic use. A framework proposed here is an effective way of managing the secondary user data transmission by keeping track of the primary user activity and also the QoS requirement of the secondary users. The QoS requirement for secondary users is managed by using trusted token analyzer. Trusted token analyzer manages the users by controlling the user data traffic.

\section{FUTURE WORK}

Cognitive Radio Network is a vast field to study. QoS requirement monitoring for the secondary user is one of the parameter we are going to focus in future. Also the primary user emulation attack is another consideration.

\section{REFERENCES}

[1]. I. F. Akyildiz, W.-Y. Lee, M. C. Vuran, and S. Mohanty,"Next generation /dynamicspectrum access/cognitive radio wireless networks: a survey," Computer Networks: The International Journal of Computer and Telecommunications Networking, vol. 50 , Issue 13, pp. 2127 - 2159, 2006.

[2]. D. Cabric, S.M. Mishra, and R.W. Brodersen, "Implementation Issues in Spectrum Sensing for Cognitive Radios," Proc. IEEE Asilomar Conf. Signals, Systems and Computers, pp. 772-776, Nov. 2004.

[3]. M.T Masonta, M. Mzyece, N.Ntlatlapa, "Spectrum Decision in Cognitive Radio Networks: A Survey" IEEE Communications Surveys and Tutorials, vol. 15, no. 3, pp. 1088-1107

[4]. L. Cao and H. Zheng, "Distributed Spectrum Allocation via Local Bargaining," Proc. IEEE Sensor and Ad Hoc Comm. and Networks (SECON), pp. 475-486, Sept. 2005.

[5]. Jarkko Paavola, “ Operational Challenges for Emerging 
Cognitive Radio Technologies -Wireless Devices Utilizing TV White Spaces", Proceeding of the 10th Conference of FRUCT Association

[6]. R. Etkin, A. Parekh, and D. Tse, "Spectrum Sharing for Unlicensed Bands," IEEE J. Selected Areas in Comm., vol. 25, no. 3, pp. 517-528, Apr. 2007.

[7]. T. Chen, H. Zhang, G.M. Maggio, and I. Chlamtac, "CogMesh: A Cluster-Based Cognitive Radio Network," Proc. IEEE Second Symp.New Frontiers in Dynamic Spectrum Access Networks (DySPAN),pp. 168-178, Apr. 2007

[8]. J. Mitola and G. Q. Maguire, "Cognitive radio: Making software radios more personal," IEEE Personal Communications, vol. 6, no. 4, pp. 13-18, 1999.

[9]. Husheng Li, Ju Bin Song, and Zhu Han; "Transient Analysis of Data Traffic in Cognitive Radio Networks: A NonEquilibrium Statistical Mechanics Approach"; IEEE Transactions On Wireless Communications, Vol. 12, No. 9, September 2013

[10]. M. Gandetto and C. Regazzoni, "Spectrum Sensing: A Distributed Approach for Cognitive Terminals," IEEE J. Selected Areas in Comm., vol. 25, no. 3, pp. 546-557, Apr. 2007. [11]. L. Cao and H. Zheng, "Distributed Spectrum Allocation via Local Bargaining," Proc. IEEE Sensor and Ad Hoc Comm. and Networks (SECON), pp. 475-486, Sept. 2005

[12]. C.-S. Chang and J.A. Thomas, "Effective bandwidth in high-speed digital networks", IEEE Journal on Selected Areas in Communications, Vol. 13, NO 6, August 1995

[13]. R. Etkin, A. Parekh, and D. Tse, "Spectrum Sharing for Unlicensed Bands," IEEE J. Selected Areas in Comm., vol. 25, no. 3, pp. 517-528, Apr. 2007.

[14]. F. Borgonovo, M. Cesana, and L. Fratta, "Throughput and delay bounds for cognitive transmissions," Advances Ad hoc Netw., pp. 179-190, Aug. 2008..

[15]. N. Nie and C. Comaniciu, "Adaptive Channel Allocation Spectrum Etiquette for Cognitive Radio Networks," Proc. First IEEE Int'1 Symp. New Frontiers in Dynamic Spectrum Access Networks(DySPAN '05), pp. 269-278, Nov. 2005.

[16]. N. Nie and C. Comaniciu, "Adaptive Channel Allocation Spectrum Etiquette for Cognitive Radio Networks," Proc. First IEEE Int'l Symp. New Frontiers in Dynamic Spectrum Access Networks (DySPAN '05), pp. 269-278, Nov. 2005

[17]. C. Peng, H. Zheng, and B.Y. Zhao, "Utilization and Fairness in Spectrum Assignment for Opportunistic Spectrum Access," ACM Mobile Networks and Applications, vol. 11, no. 4, pp. 555-576, Aug. 2006.

[18]. G. Ganesan, Y.G. Li, Cooperative spectrum sensing in cognitive radio networks, in: Proc. IEEE DySPAN 2005, November 2005, pp. 137-143.

[19]. D. Cabric, et. al., A Cognitive Radio Approach for Usage of Virtual Unlicensed Spectrum. In proc. of 14th IST Mobile Wireless Communications Summit, Dresden, Germany, June 2005.

[20]. H. Shiang and M. Schaar, "Queuing-Based Dynamic Channel Selection for Heterogeneous Multimedia Applications over Cognitive Radio Networks," IEEE Trans. Multimedia, vol. 5,no. 10, pp. 896-909, Aug. 2008.
[21]. T. Harrold, R. Cepeda, and M. Beach, "Long-term measurements of spectrum occupancy characteristics," in Proceedings of the IEEE DySPAN Conference, 2011, pp. 83-89.

[22]. B. Canberk, I. F. Akyildiz, and S. Oktug, "Primary user activity modeling using first-difference filter clustering and correlation in cognitive radio networks," IEEE/ACM Transactions on Networking, vol. 19, no. 1, pp. 170-183, Feb. 2011.

[23]. Y.-C. Kuo, "Quorum-based power-saving multicast protocols in the asynchronous ad-hoc network," Computer Networks, vol. 54, pp.1911-1922, 2010

[24]. D. Cabric, S.M. Mishra, D. Willkomm, R. Brodersen, A.Wolisz, "A Cognitive radio approach for usage of virtual unlicensed spectrum", in: Proc. 14th IST Mobile and Wireless Communications Summit, June 2005.

[25]. L. Cao, H. Zheng, Distributed spectrum allocation via localVbargaining, in: Proc. IEEE Sensor and Ad Hoc CommunicationsVand Networks (SECON) 2005, September 2005, pp.475-486.

[26]. W.-Y. Lee and I. F. Akyildiz, "A spectrum decision framework for cognitive radio networks," IEEE Transactions on Mobile Computing, vol. 10, no. 2, pp. 161-174, Feb. 2011. [27]. J. Riihijarvi, P. Mahonene, M. Wellens, and M. Gordziel, "Characterization and modelling of spectrum for dynamic spectrum access with spatial statistics and random fields," in Proc. IEEE 19th Int. Symposium on Personal, Indoor and Mobile Radio Communications, Cannes, Sep.

15-18 2008.

[28]. D. Cabric, S.M. Mishra, R.W. Brodersen, "Implementation issues in spectrum sensing for cognitive radios", in: Proc. 38th Asilomar Conference on Signals, Systems and Computers 2004, November 2004, pp. 772-776.

[29]. M. Hoyhtya, S. Pollin, and A. Mammela, "Improving the performance of cognitive radios through classification, learning, and predictive channel selection," Advances in Electronics and Telecommunications, vol. 2 , no. 4, pp. 28-38, Dec. 2011.

[30]. N. Nie and C. Comaniciu, "Adaptive Channel Allocation Spectrum Etiquette for Cognitive Radio Networks," Proc. First IEEE Int'l Symp. New Frontiers in Dynamic Spectrum Access Networks (DySPAN '05), pp. 269-278, Nov. 2005.

[31]. C. Ghosh, S. Pagadarai, D. Agrawal, and A. Wyglinski, "A framework for statistical wireless spectrum occupancy modeling," IEEE Transactions on Wireless Communications, vol. 9, no. 1, pp. 38-44, Jan. 2010

[32]. E.Z Tragos, S.Zeadally, A.G. Fragkiadakis, V. A.Siris, "Spectrum Assignment in Cognitive Radio Networks: A Comprehensive Survey", IEEE Communications Surveys \& Tutorials, Accepted For Publication 1

[33].http://www.channelpartnersonline.com/blogs/peertopeer/ 2014/01/spectrum-sharing-drives-mobile-market-growth.aspx [34].http://www.gonzalovazquezvilar.eu/cognitiveradio.htm [35]. Cognitive Radio Networks: From Theory to Practice By Ahmed Khattab, Dmitri Perkins, Magdy Bayoumi [36]. M. Cesana,F. Cuomo, E.Ekici ; "Routing in cognitive radio networks: Challenges and solutions" Ad Hoc Netwoks ELSEVIER Volume 9, Issue 3, May 2011. 
[37]. A. A. Daoud, M. Alanyali, and D. Starobinski, "Secondary pricing of spectrum in cellular CDMA networks," in Proc. IEEE Int. Symposium for New Frontiers in DySPAN, Dublin, Ireland, Apr. 17-20 2007

[38]. Norfolk State University Norfolk VA 23504 in@nsu.edu Ma Liangping ; Shen Chi Xin, Chunsheng Dept. of Computer Science. "Path-Centric Channel Assignment in Cognitive Radio Wireless Networks ". 2007.

[39]. A. Ghasemi, E.S. Sousa, Collaborative spectrum sensing for opportunistic access in fading environment, in: Proc. IEEE DySPAN 2005, November 2005, pp. 131-136. 